\title{
Unique Locomotory Mechanism of Mermis nigrescens, a Large Nematode That Crawls Over Soil and Climbs Through Vegetation
}

\author{
CARL GANS AND A. H. JAY BURR \\ Department of Biology, University of Michigan, Ann Arbor, Michigan 48109 \\ (C.G.); Department of Biological Sciences, Simon Fraser University, \\ Burnaby, British Columbia, V5A 1 S6 Canada (A.H.J.B.)
}

\begin{abstract}
Females of Mermis nigrescens, a nematode parasitic on grasshoppers, climb through terrestrial vegetation where they lay their eggs. The 100-mm-long body of these nematodes bridges gaps in this three-dimensional substratum, and crawls efficiently over planar surfaces. The nematodes do not use the classical undulant pattern of nematode locomotion as one coordinated unit; instead they propel themselves in several independent, locally controlled zones that propagate posteriorly. A repeated motion of their anterior end laces the body around fixed objects at which force may be applied. Propulsive force is applied to objects as the body glides past the contact site. Intermediate loops are elevated above the surface where they cannot contribute to propulsion. These loops rise and fall with time due to varying differences in propulsive forces between the contact sites.

Forces are applied to the objects by internally generated bending couples that are propagated along the trunk, propelling the body in a cam-follower mechanism. Bending couples are generated by the contraction of ventral or dorsal longitudinal muscle bands that apply compressive force to the cuticle. The muscle bands, consisting of a single layer of obliquely striated muscle cells, are closely applied to the cuticle and are separated from it only by a fibrous basal lamina and a thin extension of a hypodermal cell. The myofilaments of each sarcomere are parallel to the body axis and attached perpendicularly via dense bodies (z-line equivalents) to the basal lamina, which in turn is fixed to the cuticle via filaments passing through the hypodermal cytoplasm. Consequently, forces are transmitted laterally to the cuticle over the entire length of the muscle, compressing it parallel to the surface without need for attachment to the terminal ends of the muscle cells. Thus the muscles are engineered for local control of bending and avoidance of buckling. There is evidence that the motor nervous system of Mermis may not be as simple as in classical nematode examples, which may explain why Mermis is capable of a much more localized control of locomotory motion. 1994 Wiley-Liss, Inc.
\end{abstract}

After mating, the nonfeeding female of the parasitic nematode Mermis nigrescens lies dormant in the soil for almost 2 years while her eggs develop. Then, during wet weather, she migrates to terrestrial vegetation on which she lays her eggs (Cobb, '26, '29; Christie, '37). Unlike the well-studied nematodes Caenorhabditis elegans and Ascaris suum, adult female Mermis do not move by the classical undulant pattern (Gray and Lissmann, '64). Instead, they locomote with a variable, puzzling wave motion that has never been analyzed or described, although other nonclassical locomotory patterns of nematodes have been noticed (Stauffer, '24; Streu et al., '61; Reed and Wallace, '65; Croll, '70; Crofton, '71; Lee and Atkinson, '77).

The movements involved may be compared not only to those of typical nematodes, but also to those of elongate aquatic and terres-

\footnotetext{
Address reprint requests to Carl Gans, Dept. of Biology, University of Michigan, Ann Arbor, MI 48109.
} 
trial vertebrates (Gans, '86). The comparisons must take into account the architectures of the internal force production and transmission systems. Nematodes transmit forces to external objects via a thick cuticle. The obliquely striated muscles of nematodes, described previously (Rosenbluth, '65, '67; Hope, '69; Valvassori et al., '81; Waterston, '88), must somehow link longitudinal forces generated by the contractile filaments to the local cuticle.

As important as architecture are comparisons of scale. Not only do vertebrates commonly have diameters at least an order of magnitude greater, but at a diameter of 0.4 $\mathrm{mm}$ and a mass of $15 \mathrm{mg}$, Mermis falls into a size range in which capillary adhesion effects can be significant (Denny, '93). The length-todiameter ratio of the nematode is $\sim 300$ (total length $\sim 120 \mathrm{~mm}$ ), much greater than that of vertebrates and typical nematodes. In Ascaris, the ratio is 50 (Johnson and Stretton, '80). Evidently nematode architecture must somehow overcome the tendency for a long narrow tube to buckle under longitudinal compression.

This report describes the basic motor architecture and locomotor patterns of Mermis. It stresses kinematics, but also provides information about the production of internal forces and the nature and sites of their exertion onto the environment. It attempts to match the peculiar morphology observed with the functional differences seen in this species.

\section{MATERIALS AND METHODS}

Gravid female Mermis nigrescens, available once per year, were collected in the Fraser Valley near Vancouver, British Columbia, Canada, and maintained in their dormant state in soil in the dark at temperatures of $10^{\circ} \mathrm{C}$. Others were removed from artificially infected Shistocerca gregaria hosts at full size and maintained in soil at greenhouse temperatures until molted to adult females and eye color was just visible, $\sim 4$ months. Prior to behavioral observations, collected Mermis were transferred to a humid chamber with horizontal illumination and the temperature was raised to $21^{\circ} \mathrm{C}$. Normally, 5-20\% of worms can be activated on a given day under these conditions.

Animals were videotaped using several standard cameras with a macro lens at $30 \mathrm{fps}$ at various magnifications and angles while the worms were traveling horizontally over the surface of black felt that was intermittently sprayed with distilled water (Burr et al., '90). This study is based on $\sim 14$ hours of VHS and $8-\mathrm{mm}$ videotape, plus additional direct observations. The tapes were analyzed and figures prepared using a Panasonic AG 6300 single frame video analyzer. Some specimens were allowed to climb vertical surfaces (e.g., felt, glass panes, and chicken-wire mesh). Movements on these substrates were compared with videotapes of travel over the surface of the soil, as well as amid grass and other vegetation in the field and in the laboratory. The sites and magnitudes of force application were established by having Mermis cross gaps in the substrate from the main body of felt to and from a patch of felt that was suspended from a sling, for which the force required to achieve a unit of displacement could be calibrated using Pesola scales. The continuous application of force in grass was monitored by video recording the deflection of grass blades.

Some of the anatomical description derives from preparations made earlier for other studies (Burr and Harosi, '85; Burr et al., '89; Burr and Babinszki, '90). Specimens prepared for transmission electron microscopy (TEM) were killed instantaneously by immersion in cold $3 \%$ glutaraldehyde in $0.05 \mathrm{M}$ phosphate buffer at $\mathrm{pH} 7.2$, cut into $1-\mathrm{mm}$ pieces, and fixed $\sim 16 \mathrm{hr}$ at $5^{\circ} \mathrm{C}$, then washed in buffer. They were further fixed in $2 \%$ OsO4 in the same buffer for $2 \mathrm{hr}$, then washed in buffer. They were transferred through an ethanol series to propylene oxide and embedded in Araldite: 1 day in a 50\% mixture with propylene oxide and 4 days in $100 \%$. The Araldite mixture contained Araldite 6005 (37.5\%), RD-2 (12.5\%) and dodecenyl succinic anhydride (50\%), and DMP-30 accelerator $(1$ drop per $5 \mathrm{ml}$ ). After polymerizing at $65^{\circ} \mathrm{C}$ and remounting, silver-gray transverse or longitudinal sections were cut on a Reichert Om U2 ultramicrotome with a diamond knife and mounted on uncoated copper grids. These were stained $15 \mathrm{~min}$ in $5 \%$ uranyl acetate and $5 \mathrm{~min}$ in lead citrate and observed in a Philips 300 electron microscope at $80 \mathrm{kV}$. As well, $1-\mu \mathrm{m}$ sections were photographed under phase illumination. Several specimens of Mermis were fixed in 3\% paraformaldehyde, wax embedded, and crosssectioned at $7 \mu \mathrm{m}$. They were stained either with hematoxylin or with DAPI $\left(4^{\prime}, 6^{\prime}\right.$-diamidino-2-phenylindole $\mathrm{HCl}$ ), a fluorescent DNA stain. In addition, wholemounts were observed by differential interference contrast or fluorescence illumination to demonstrate commissures and nuclei of the motor ner- 
vous system. Muscle cell length was measured in polarized light micrographs.

\section{RESULTS \\ Morphology}

Mermis is very slender and outwardly cylindrical. The length and diameter of female specimens range from 50 to $150 \mathrm{~mm}$, and $.25-.5 \mathrm{~mm}$, respectively and their mass is 5-15 mg just before the start of egg deposition ( $N \geq 20$ ). Normally smooth, the surface of desiccating animals forms accordionlike rings, possibly suggesting that normal body form reflects internal hydrostatic pressure. Similar corrugations are observed on the inside of sharp bends. As is true for Ascaris (Harris and Crofton, '57), transection of the Mermis body produces immediate discharge of fluid and release of pressure.

\section{Cuticle}

The transparent cuticle is extremely thick, measuring $1 / 5$ th $-1 / 3$ th of the radius of the nematode (Fig. 1). The typical nematode cuticle is $1 / 15$ the radius (Bird and Bird, '91). Lacking the external lateral ridges (alae) observed in other nematodes, the cuticle is markedly thickened along inward lateral ridges, as is typical of mermithids (Kaiser, '91). The structural function may be like that of alae, to stiffen the body against lateral bending. In a region of the head in which small radius lateral bending is known to occur during scanning motion (Burr et al., '90), these ridges are diminished. The ridge is present in the $2-\mathrm{cm}$ neck region behind the head where larger radius lateral bending occurs as well as in the rest of the body where lateral bending is not observed.

The nematode cuticle is basically a threelayered cylinder composed of various crosslinked collagens and covered by a thin epicuticle of another material (Wharton, ' 86 ; Wright, '91; Bird and Bird, '91). The cuticle of Mermis, described by Lee ('70), is shown in transverse section in Figure 2. The osmiophilic, 10-nm epicuticle is not resolved at this magnification. The outermost layer (cortical zone) is composed of a homogeneous-appearing material penetrated by radial canals through which electron-dense material of the surface coat is secreted. Next, the median zone is a spongy material through which two layers of large, oval "giant" fibers wind helically around the body with opposite handedness. The crossing angles range from $111^{\circ}$ to $122^{\circ}$ in wholemounts. Innermost is the thick

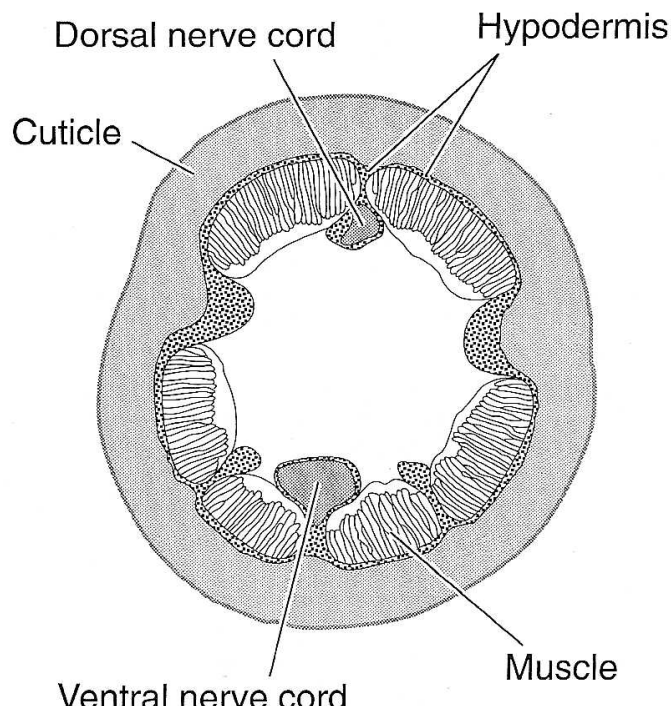

Ventral nerve cord

$$
50 \mu \mathrm{m}
$$

Fig. 1. Mermis nigrescens. Cross section showing structure of the body wall and location of neurons. A thin extension of hypodermal cells separates the cuticle and longitudinal muscle bands. Interneurons and ventral motor neurons are contained within the ventral cord; dorsal motor neurons originate in the ventral cord and send their axon or dendrite to the dorsal nerve cord via commissures (not shown). From a nearly transverse section.

basal zone containing many layers of smaller fibers (Fig. 2). In longitudinal sections these are seen to travel longitudinally before bending toward a radial orientation and interdigitating with other fiber layers (Lee '70; Burr, unpub.).

\section{Muscles}

Nematode body-wall muscle is longitudinal and is separated from the cuticle only by a basal lamina and a 1.5- $\mu \mathrm{m}$-thick extension of the hypodermis (Figs. 1-3). The longitudinal muscle bands lie in subdorsal and subventral quadrants separated by cords of the hypodermis. The dorsal quadrants of Mermis and other mermithids (Kaiser, '91) are unusual in having only a single muscle band; the ventral quadrants have the normal two on each side (Fig. 1). The greater cross-sectional area of muscle in the ventral quadrant suggests that there is more force available for ventral than for dorsal bending.

In adult female Mermis nigrescens, the arrangement of the muscle cells and contrac- 


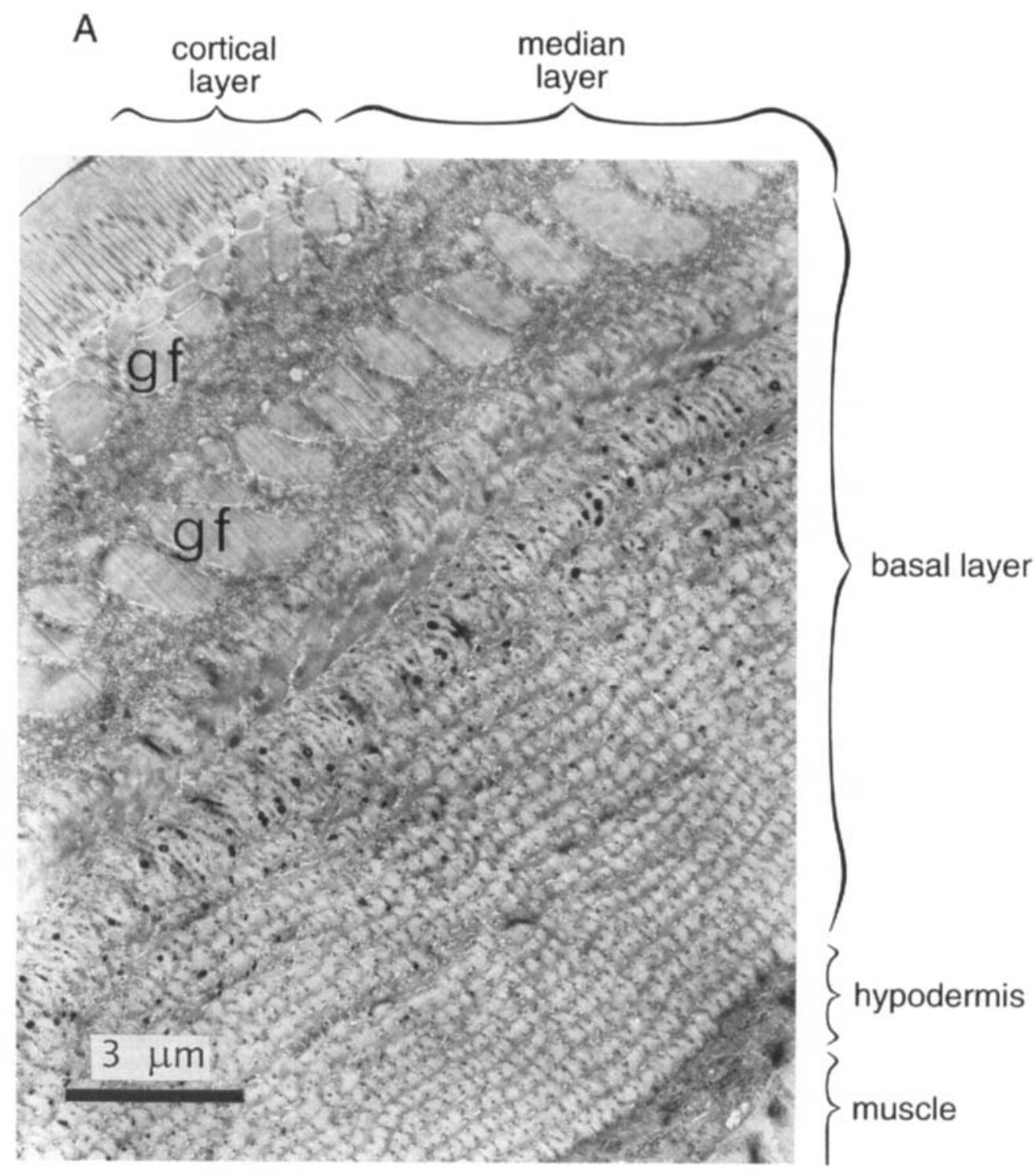

Fig. 2. Mermis nigrescens. Transverse section showing structural zones of the cuticle. gf, giant fiber. Transmission electron micrograph.

tile apparatus within the muscle bands is very similar to the pattern in other large nematodes, e.g., Ascaris (Rosenbluth, '65, '67). This is classified as polymyarian (more than five longitudinal rows of muscle cells in each band) and coelomyarian (having the contractile region of each cell folded longitudinally to form two parallel radial plates) (Hope, '69; Bird and Bird, '91). In Mermis, each band is composed of 5-30 rows of narrow, ribbon-like cells surrounded by basal lamina
(Figs. 1, 3). The roughly 2 -mm-long, $2-\mu \mathrm{m}$ wide, cells overlap obliquely at their longitudinal ends and the myofilaments are joined by half-desmosome connections to the basal lamina separating them. Similar observations are reported in larvae of an unidentified Mermis species (Valvassori et al., '81).

The radial plates of longitudinal contractile filaments are closely apposed to the plasma membrane and the basal lamina; as described below, the myofilaments are con- 


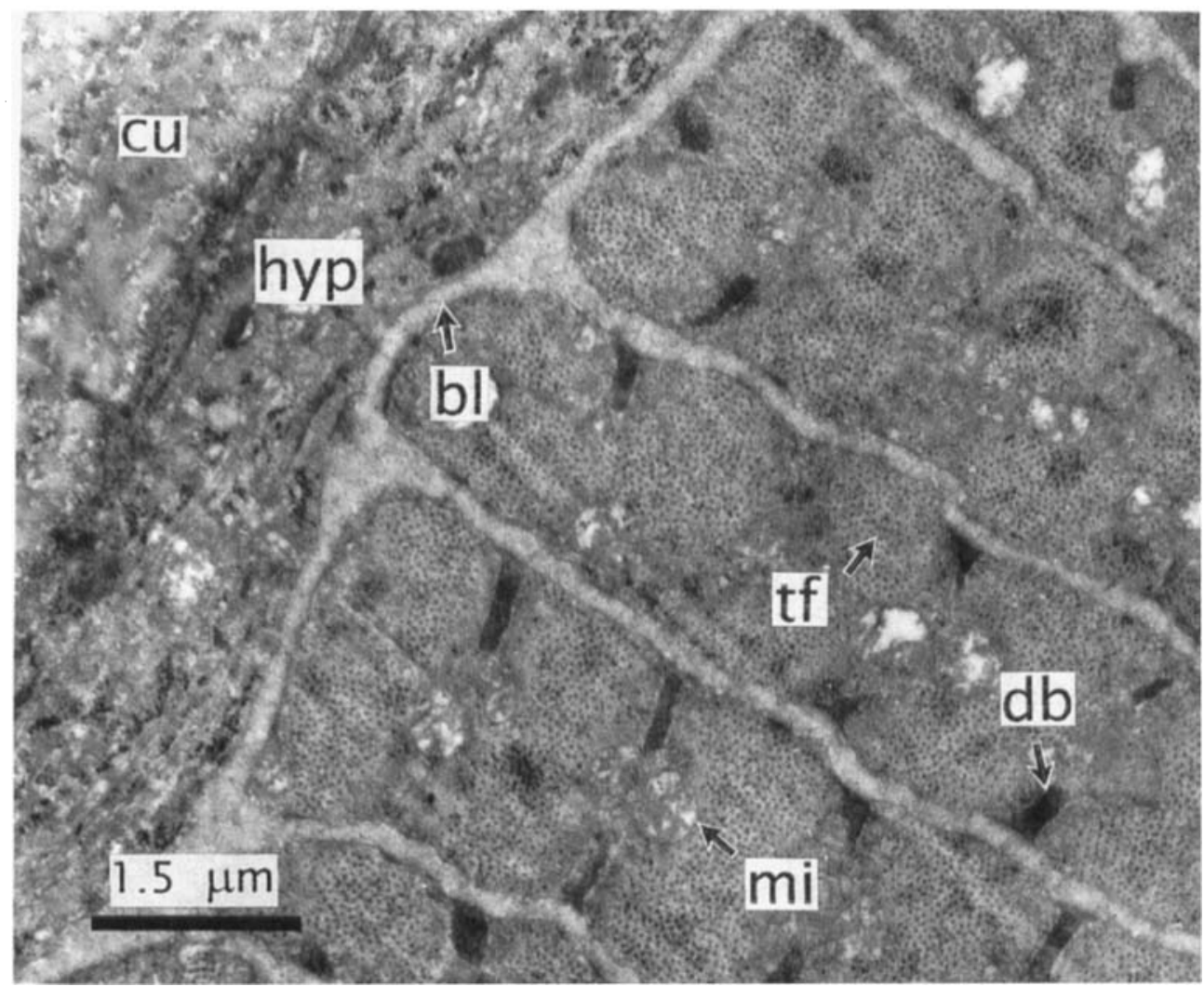

Fig. 3. Mermis nigrescens. Transverse section of body wall muscle, basal lamina and hypodermis. bl, basal lamina; cu, cuticle; db, dense body; hyp, hypodermis; mi, mitochondrion; tf, region containing thick myofilaments.

nected perpendicularly to the plasma membrane via dense bodies (Fig. 3). The fibrous basal lamina between the cells is continuous with the basal lamina enclosing the muscle band (Fig. 3), which, in turn, is connected across the thin hypodermal cytoplasm to the cuticle at closely-spaced intervals via halfdesmosomes and intermediate filaments (Francis and Waterston, '91). Thus, this contractile apparatus, as in other nematodes, differs fundamentally from that of vertebrates by being linked to the cuticle all along the length of the muscle cell, rather than only at its ends.

Unlike cross-striated muscle of vertebrates, nematode locomotor muscle cells are obliquely striated (Toida et al., '75; Lanzavecchia, '77). The myofilaments lie parallel to the long axis of the worm. However, in any radial plane, adjacent myofilament rows are offset longitudinally. Their attachment to dense bodies, cytoskeletal components equivalent to Z-lines of cross-striated muscle, are similarly offset, making the sarcomere arrangement appear parallelogramic in radialplane sections through the contractile apparatus. The ends of the filaments in adjacent radial planes are aligned along a perpendicular to the filaments which is also normal to the surface of the radial basal lamina sheath between the cells. The ends of the actin filaments are connected along this direction to the sarcolemma by dense bodies. These are tied across the sarcolemma to the basal lamina. Another cytoskeletal structure (Mline analog), similarly links the centers of the thick filaments to the cell membrane and basal lamina (Waterston, '88). The basal lamina is structured as if to transmit forces parallel to its surface. Fibrous strands run in multiple directions in the lamina (Rosenbluth, '67) and evidently are attached across 
the sarcolemma to the intracellular dense bodies (Francis and Waterston, '91).

In the coelomyarian type of nematode muscle, these attachments connect the contractile filaments perpendicularly to the radial sheath between cells, then radially to the cuticle. Local contraction shortens the distance between dense bodies and the force is transmitted radially and probably obliquely via the fibrous basal lamina sheath to shorten a local region of the attached cuticle. Thus, contractile forces are applied to the cuticle locally and continuously along each muscle band, rather than only at the ends of the muscle fibers as in vertebrates and arthropods. In Mermis, as in Ascaris (Rosenbluth, '67), localized compression of the cuticle can produce an accordion-like, $500-\mu \mathrm{m}$ corrugation in its outer surface. This is to be distinguished from the fine, $0.33-\mu \mathrm{m}$-spaced annulations resolved by the electron microscope in the cuticle of Mermis (Lee, '70; Burr, unpublished) and other nematodes and also thought to provide flexibility (Bird and Bird, '91).

\section{Motor system}

The muscles in the dorsal and ventral quadrants of the nematode body wall are controlled by motor neurons in the dorsal and ventral cords respectively. Each muscle cell sends a process to the nearest nerve cord where it receives excitatory and inhibitory innervation (Rosenbluth, '65; Stretton, '76; White et al., '76; Walrond et al., '85). The cell bodies and nuclei of both the dorsal and ventral motor neurons lie in the ventral cord, and some send an axon or dendrite to the dorsal cord via circumferential commissures (Stretton et al., '78; Johnson and Stretton, '80; White et al., '86). This pattern also occurs in Mermis: Nomarski differential interference contrast discloses commissures under the transparent cuticle and DAPI fluorescent staining shows no nuclei in the dorsal cord. Unlike Caenorhabditis elegans and Ascaris, numerous ( $80 / \mathrm{mm}$ ) large hypodermal cell nuclei are located in the ventral cord (Fig. 4) and the numbers of commissures are uniquely high. This prevented the enumeration of motor neuron nuclei. On the other hand, we counted 11.6 commissures per $\mathrm{mm}$ in one worm, suggesting a total of approximately 1,000. In Caenorhabditis and Ascaris, seven out of 11 motor neurons send commissures to the dorsal cord (White et al., '76; Stretton et al., '78); thus the number of motor neurons in Mermis must be more than a thousand. As yet we have not succeeded

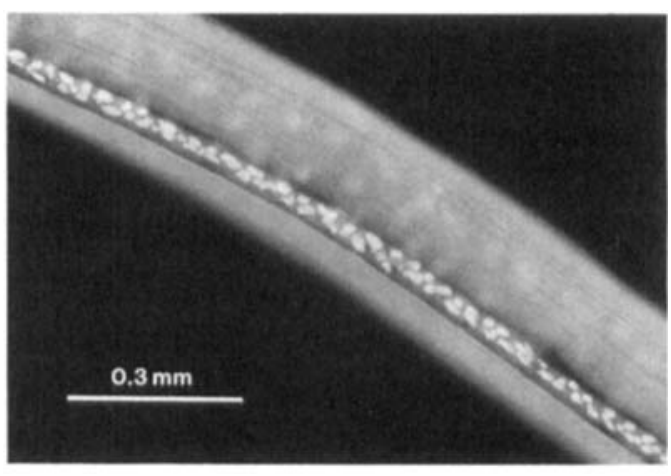

Fig. 4. Mermis nigrescens. Distribution of nuclei along ventral cord. Ventral view of DAPI-stained, glutaraldehyde-fixed wholemount of a region anterior to the vulva.

identifying sensory neurons by light microscopy.

In both Caenorhabditis elegans (White et al., '76, '86) and Ascaris suum (Stretton et al., '78) the motor neurons of the ventral cord are arranged in five units, each with a basic set (ignoring the extra neurons in the anteriormost units) of 11 cells and seven commissures. There are no sensory neurons in either the dorsal or ventral cords. In adult hermaphrodites of Caenorhabditis, 57 motor neurons with 36 commissures innervate 79 muscle cells, and in adult females of Ascaris, 59 motor neurons with 36 commissures innervate $\sim 50,000$ muscle cells (Stretton, '76; Sulston and Horvitz, '77). By comparison, the total number of muscle cells in adult female Mermis is $\sim 10,000$ (length of each muscle cell, $2 \mathrm{~mm}$; number per cross section, 200 ; and length of the worm, $100 \mathrm{~mm}$ ). The number of muscle cells appears to correspond to the length of the nematode, but the number of neurons clearly does not. The much larger motor system of Mermis may be related to the more complex locomotory behavior we observe.

\section{Movement patterns \\ Locomotion}

The travel of Mermis over horizontal surfaces proceeds by a unique combination of body movements that are most clearly revealed in video recordings of locomotion on moist felt, such as the sequence shown in Figure 5. The crawling motion appears sluggish. Movement between contact sites requires six or more seconds and movement of one $100 \mathrm{~mm}$ body length requires $\sim 60 \mathrm{sec}$ in an active individual crawling effectively on 


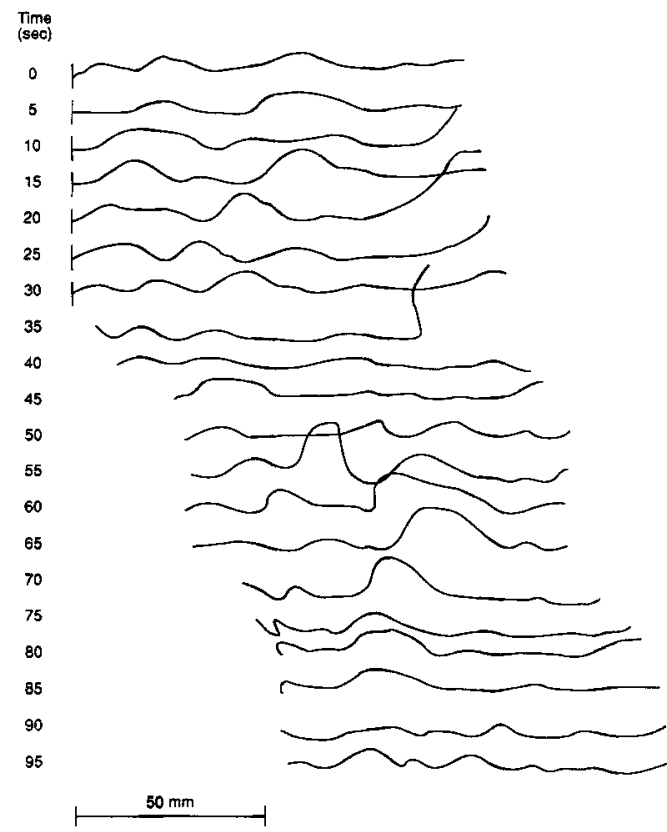

Fig. 5. Mermis nigrescens. Body wave fluctuations in a side view. Tracings from video recordings during phototaxis on moist felt surface. Sequence begins with tail out of the field of view to left.

felt (Burr et al., '90). The locomotion illustrated in Figure 5 is not that of Thomas, '90). Note, however, that Mermis adults neither feed nor defecate; they live on food stored during a parasitic larval stage and lack an anus.

Typically, during steady locomotion, $\mathrm{Mer}$ mis contacts a flat horizontal surface at 6-8 ventral points with an elevated wave between each point (Burr et al., '90). At any instant, the curves formed by the body are stiff; they are conserved for a short time whenever a portion of an animal is pushed or lifted from the surface.

Observation of the movement of the body trunk through the curved trajectory is facilitated by the obvious dark egg masses and the vulva visible through the transparent cuticle, e.g., in the locomotion sequence on felt represented by Figure 6 . Over periods of $2-4 \mathrm{sec}$, the position of the bends remains stationary in space while the body flows through the bend. Thus over short times, the wave or loop of body created between two contact points establishes an aerial trajectory through which all of the following portions of the body travel. The body even passes through irregularities in the curvature of the elevated portions be-

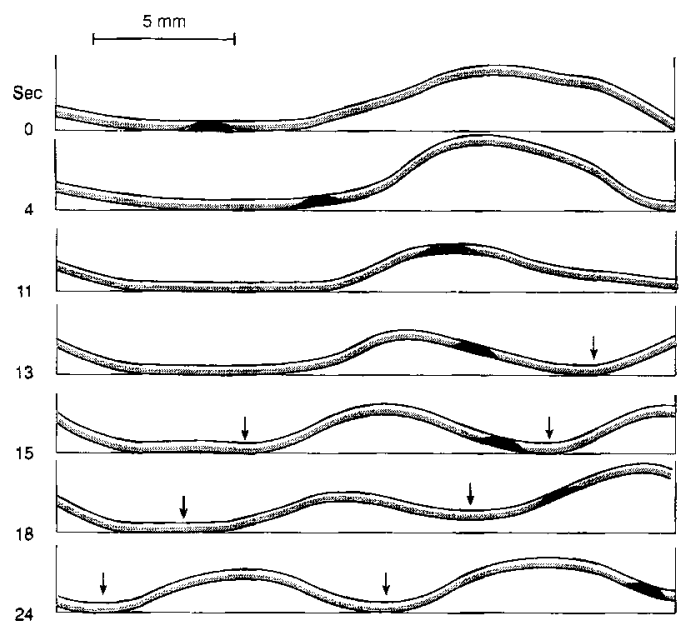

Fig. 6. Mermis nigrescens. Propagation of bends along body, slow change in wave shapes, and slippage of contact points along surface (arrows). Tracings from video tape of side view during phototaxis on felt surface. The position of the vulval region, shown in solid black, indicates the motion of the trunk through the wave trajectory.

tween contact sites. The motor system of Mermis must be capable of accurately propagating a local curved region along the body as it moves past a point in space.

Video recordings indicate that the shape and sizes of the waves between the fixed lacing sites change gradually (Figs. 5,6). Whenever the worm travels concertedly toward a particular goal such as a light source, the vertical waves between contact points show minimal elevation as in Figure 5, secs 0 , 40, 90-95. At other times, these portions between contact sites can gradually form large vertical and horizontal waves, which sometimes twist to one side or fold into helical loops. We show below that the various elevated wave shapes are not directly related to propulsion, but are a consequence of differing propulsive velocities at the ventral contact points.

Locomotion has to be analyzed in terms of various movement routines. Key among them are lacing, head elevation bouts including scanning and orientational motions, and nictating. Reverse locomotion is also observed. The subroutines not only establish the sites of force transmission, but also establish the path that will be traversed by the animal.

Lacing and other contact sites

A worm progressing over a horizontal felt surface terminates a head elevation bout with 
a unique lacing motion of the anterior few millimeters of the body. It pokes the anterior tip into the substrate (Fig. 7a), then slides the tip along the surface in a hooking motion (Fig. $7 \mathrm{a}-\mathrm{c}$ ) that is actually helical. This frequently succeeds in lacing the body under one or more fibers on the surface of the felt. After the head advances forward from the lacing site, the body just emerging from the site bends upward as the head is elevated, thus forming a tight curve at the site (Fig. $7 \mathrm{~d}-\mathbf{f}$ ). The increased curvature at the lacing site is maintained as the body glides through the site. This is shown below to be instrumental in the application of a propulsive force to the fiber. The head remains elevated until the next lacing site is probed. Lacing is more successful on felt than on the many other surfaces observed and tested; however, on other surfaces the general pattern is equivalent.

Movement patterns in an array of threedimensional surfaces such as encountered in grass is more complex, but with the observations on planar surfaces analyzed, they can be readily understood. For example, in the sequence illustrated in Figure $8 \mathrm{a}-\mathrm{c}$, one can recognize lacing and tight curve formation about a grass blade. As well, the bending of the grass blade indicates the concomitant application of force.

Propulsive forces are strongest when generated at the lacing sites as these are the most secure positions of contact with the substrate. Whenever the substrate makes lacing difficult, the animals appear to propel themselves by use of other contacts with surface irregularities. On moist surfaces including felt, soil and grass, advantage is often taken of adherence by surface tension at con-

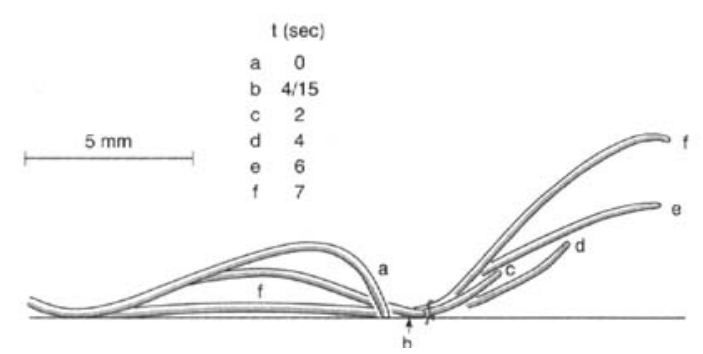

Fig. 7. Mermis nigrescens. Lacing sequence and beginning of head elevation bout on felt. (a) poking into surface; (b) sliding along surface; (c) just after lacing body under surface fiber; (d,e,f) head elevation with anterior tip scanning direction of light source. Tracing from videotape of phototaxis.

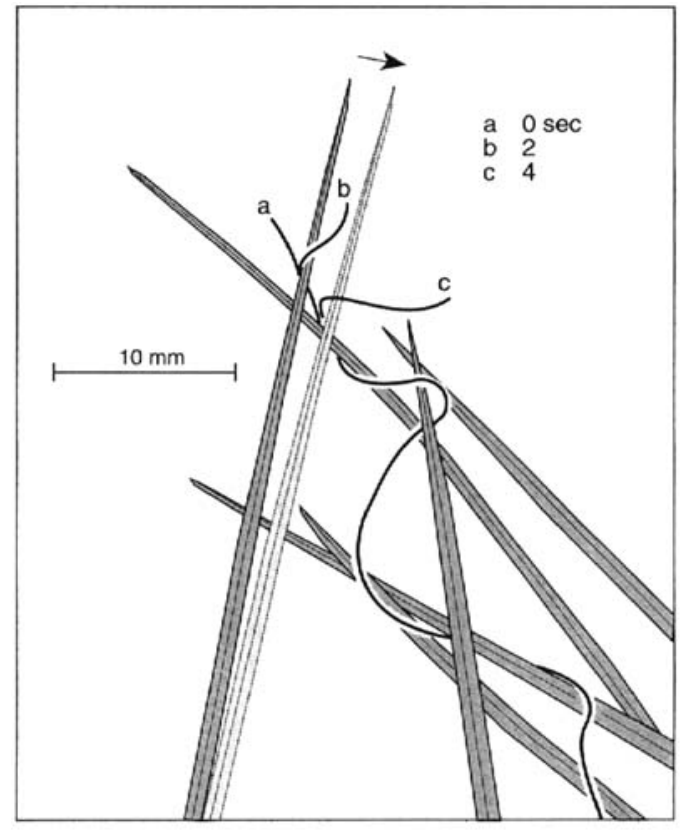

Fig. 8. Mermis nigrescens. Lacing sequence and force application while climbing through grass. (a) Touching grass blade; (b) lacing around blade. (c) force application bending blade to right. Tracing of anterior portion from videotape. Movement of grass blade (arrow) is not to scale.

tact points or zones along the body. Whenever lacing is not possible, the velocity is lower and one commonly sees slippage at the zones of contact with the substratum (Figs. $5,6)$.

\section{Scanning and orientation}

A head elevation bout begins with the emergence of the head from a lacing site (Fig. 7c) and continues until the next contact with a surface. Throughout the head elevation bout, the anterior $2 \mathrm{~mm}$, or "head" of the worm, oscillates in a scanning motion involving both vertical and sideways swinging motions (Fig. $7 \mathrm{~d}-\mathrm{f}$ ). The next 10-20 mm, or "neck," are involved in orientation to light or gravity (Burr et al., '90). The scanning motion of the head modulates the photoreceptor illumination during phototaxis, and it is independent of the orientational motion of the neck (Burr and Babinszki, '90). Orientation of the neck establishes the direction of locomotion and is modulated by the sensed direction of gravity or light (Burr et al., '90). The neck can be bent sideways or in the vertical plane. The neck can also be involved in generation of propulsive forces; however, the body behind 
the neck is primarily involved in locomotion and only dorsoventral bending is observed (Burr et al., '90).

\section{Reversing}

Nematodes commonly reverse locomotion, thereafter turning and resuming forward movement. Caenorhabditis elegans reverse spontaneously every $90 \mathrm{sec}$ on average (Burr, '85) and reversal is elicited within 2 sec after touching the anterior end, or more weakly by light and changes in temperature, osmolarity, or concentration of odorants (reviewed by Chalfie and White, ' 88 ). The neural circuit for the touch reflex is well worked out for Caenorhabditis elegans at the level of identified nerve cells (Chalfie et al., '85; Rankin, '91).

Reversing is observed occasionally in $\mathrm{Mer}$ mis, but it nearly always occurs following the cessation of light during phototaxis. Described initially as a stop response (Burr et al., '90), full reverse locomotion has now been observed as a continuation of this response. Remarkably, it only starts some 11-30 sec after the light source has been turned off. It begins with the halt in forward locomotion, then, the body simultaneously forms a series of waves of shorter wavelength than usual-the "stop" response observed previously. This simultaneously involves the entire body, rather than successively localized regions, as in forward locomotion. Then, reverse locomotion begins. Unlike that in Caenorhabditis elegans, the tail does not move as fast as the body. As a result the body coils as it moves posteriorly. After $\sim 60 \mathrm{sec}$, the forward locomotion resumes, but in a different direction than previously.

\section{Nictation}

Nictating behavior might be deemed a locomotor subroutine. In Mermis it facilitates navigation through a three-dimensional array of surfaces, such as the terrestrial vegetation on which it lays its eggs. Nictation occurs whenever $>12$ sec elapse without the head coming into contact with a surface. This can occur during a head elevation bout when crawling on soil or when encountering openings in vegetation. Nictation consists of wide, horizontal, and circular swings of the anterior body. As the body is propelled past the anteriormost contact point, a continuously increasing portion of the animal, up to half its length, becomes involved in the swinging. Nictation terminates whenever the swinging anterior portion contacts another object, such as a grass stalk, whereupon the worm tends to bend or lace here and locomotion transfers onto the new site. Nictation when crawling on soil makes it possible to discover plants and other objects that may be climbed. However, their selection is by chance collision during the process of nictation. Nictation similarly facilitates the discovery of new surfaces after encountering an opening in vegetation. A nictation bout can terminate in a collision with the previous surface- the usual case on a horizontal surface such as soil or the experimental felt.

Whenever the nictating portion of the animal makes a contact, it commonly tends to start a very different body orientation and the subsequent movements indicate the independence of different regions of the trunk. The elevated body anterior to the new contact site reorients, bending sharply in the region of contact while retaining its previously existing curves anterior to the bend These remain frozen in position for a while, although the more posterior portions of the body keep on flowing through the new bend, propelled by forces generated at posterior lacing sites. The new contact site and bend can be made anywhere along the reach of the nictating portion whereupon additional propulsive forces may develop at these sites. If the anterior tip makes contact, a new lacing site may be formed by a normal lacing bout. The various movement routines in different body regions appear independent, which is completely untypical of classical nematode locomotory motion.

\section{Evidence of cantilevering}

Several observations show that Mermis is capabie of cantilevering - elevating its anterior or posterior body above the substrate by applying a torque at the nearest contact, which acts as the fulcrum. For example, when the anterior tip is elevated during a head elevation bout (Fig. 7d-f), contraction of dorsal muscles in the region of contact would cause upward bending and thus provide the torque, whereas the posterior body is held down by laced regions, gravity, or surface tension. Another example occurs during phototaxis to a light source elevated by $45^{\circ}$ above a horizontal felt substrate (Burr et al., '90), Remarkably, the anterior portion is held oriented toward the source while the body glides through the anteriormost contact point and, as a result, as much as a third of the body becomes cantilevered and aimed at the source. Nictation is an extension of this example, 
with an extensive length of the body not only suspended anterior to a contact, but also being rocked and twirled. What is striking in all of these cases is the ability of the region in which torque is developed to be propagated posteriorly as the body glides past the fulcrum. The activity in muscles and local motor neurons is continually replaced by equivalent activity in adjacent posterior regions.

\section{Generation of propulsive force}

The lacing sites clearly are the primary sites of force transmission, as documented by several incidental observations. On felt, the caudal portion of a Mermis commonly may be seen suddenly to accelerate away from the restraint of a surface fiber whenever the caudal tip passes out from under the lacing site. This movement must have reflected the stress incorporated in the last loop. This is seen even more graphically whenever the fiber of a newly formed lacing site suddenly ruptures, for the anterior tip then makes a sudden upward motion (Fig. 9). The upward force would appear to be due to torque transmitted along the body from the posterior cantilevering site. The tension developed by contraction of the dorsal muscle bands during cantilevering is evident. As the tension is relieved, $1 / 15 \mathrm{sec}$ after the break, the curvature of the regions anterior and posterior to the fulcrum changes in the manner expected if upward bending couples had been present in these regions.

Whenever Mermis does not manage to lace under a fiber at a contact point, it will still form a downwardly bent profile and touch the surface but the contact point is observed to slip backward with time. This suggests that if a fixed site had been secured, the body

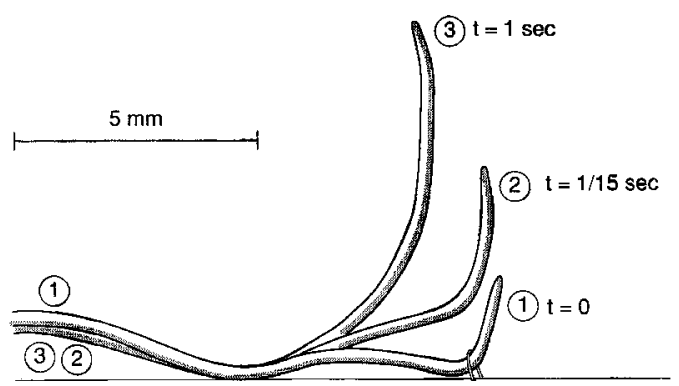

Fig. 9. Mermis nigrescens. Evidence of force application to a lacing site on felt and of cantilevering at a more posterior contact point. 1, force being applied to fiber prior to breaking; 2, release of tension just after fiber breaks; 3 , further dorsal flexure. Note changes in curvature. Tracings from videotape of phototaxis. would have exerted a propulsive force at that site.

The application of forces also was monitored by having worms travel from a fixed surface to a floating or suspended platform, the excursion of which is very sensitive to horizontal force applications. Mermis traversing from lacing sites on the fixed substratum to new ones on the floating platform exerted horizontal forces of $<0.1 \mathrm{~g}$, the minimum detectable by our force transducer. These forces or deflections occurred irregularly with differing amplitudes and at differing rates. Apparently the stiffened body transmits forces between the lacing sites. In field videos of Mermis climbing amid grass blades, similar force application is seen to cause variable but continuous deflection of a grass blade while the body slides past the blade (Fig. 10). The sequential inserts show that, during force application, the large curvature of the body in the region of the contact point is maintained while the body anterior to this region is waved about independently, probably in search of a new lacing site.

In closeups of lacing sites on felt where one can discern the threads under which the worm looped, one can see these threads being stretched and can determine the times and vectors along which forces are being applied. The force application appears to be continuous. Whenever the worm applies substantial forces, its curvature along the lacing site has a characteristic shape; its radius is lowest just anterior to the contact with the fiber and much greater posterior to the fixed site (Fig. 9).

\section{Surface tension effects}

Mermis are small enough to be affected by surface tension, and this affects their propulsive patterns. For instance, if tossed against a vertical plastic mesh that has been sprayed with water, the body will adhere to it without slipping. The body is smooth and naturally slippery, not sticky; therefore, the adherence must be due to the forces of surface tension. The forces of surface tension are sufficient to maintain the 5-15-mg animal in the vertical plane and to facilitate its vertical locomotion. Whenever the body contacts a wet grass blade during nictation, it obviously sticks to it. The body adheres to plant stems at right angles to it. When a portion of the worm contacts a portion of the sprayed felt, one can then see deformation of the droplet meniscus at the contact site, indicating that forces can be transferred between the body and the surface film. 


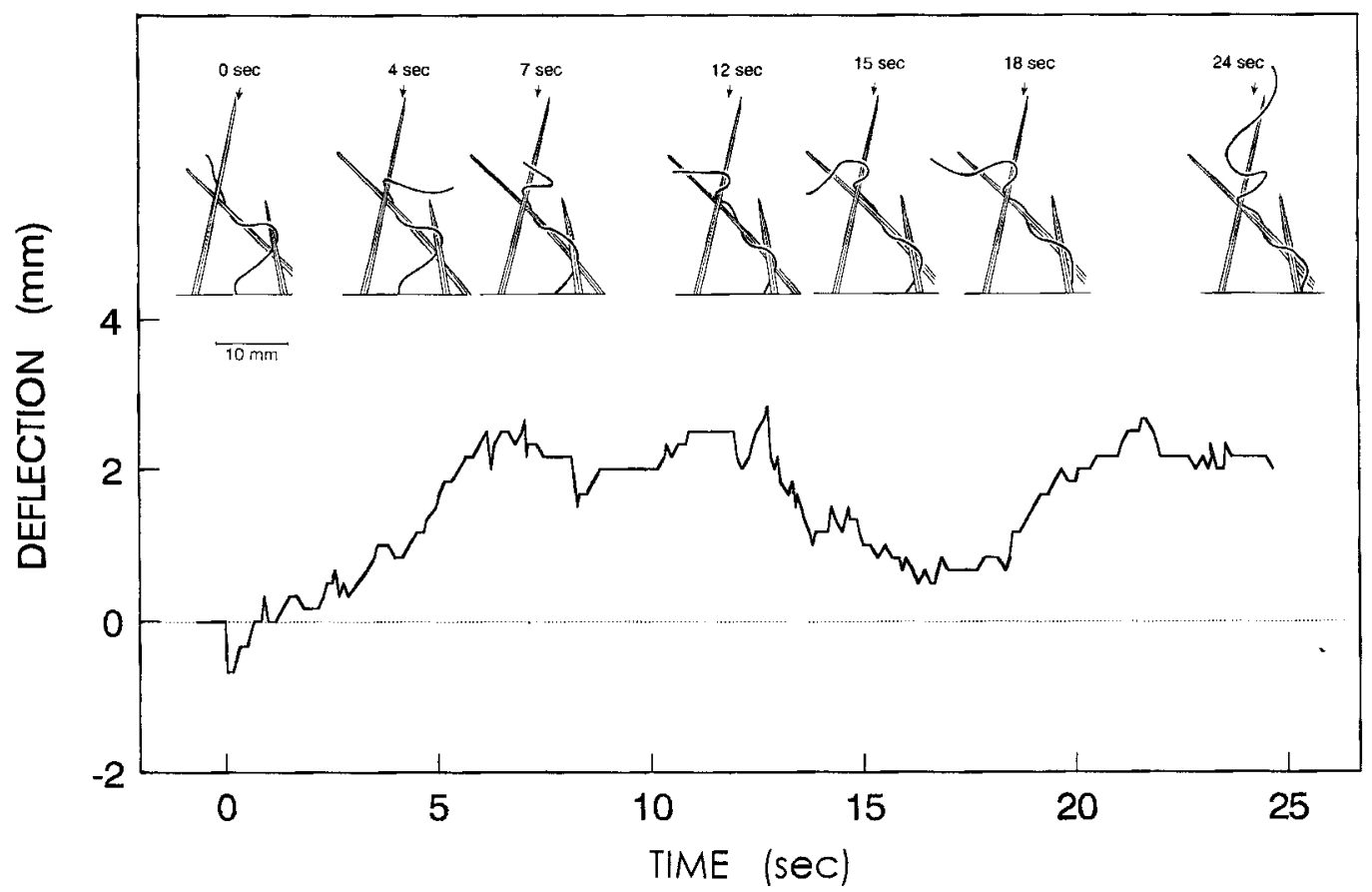

Fig. 10. Mermis nigrescens. Application of force to a lacing site in grass. Inserts: Sequential body positions and deflection of grass blade in side view. Tracings from videotape. Arrows indicate original blade position. Graph: Deflection of grass blade maintained during sliding contact with blade.

A similar force transfer occurs whenever the animals traverse smooth planar surfaces, such as wetted vertical glass or broad leaves. In such cases the body waves are flattened against the surface by the action of surface tension, although the head and neck are repeatedly raised away from the surface and locomotion is slower and less effective. Such film-embedded animals show at least two movements. The first is slippage of the flattened body waves over the surface, primarily in the anterior half. In the second, the posterior body breaks away from the surface and is placed into a more advanced position. On vertical wetted glass, the second motion can be more effective in gaining altitude than the first. In both cases, propulsive forces are generated by pushing against the resistance of surface tension, sliding friction and/or plant hairs.

\section{DISCUSSION \\ General}

Nematoda are divided into two major groups representing two independent clades: Mermithidae are included in the class Adeno- phorea; Ascaris and Caenorhabditis belong in the subclass Rhabditia of the class Secernentea (Maggenti, '83). Adult Mermis resemble Nematomorpha, which also have an extremely elongated, free-living, nonfeeding adult stage (Hyman, '51). There are similarities in the cuticle, particularly the presence of helical crossed-fiber layers (Zapotsky, '71; Cham et al., '83; Seymour, '83), and in the body wall muscle, with the coelomyarian muscle structure and plate-like muscle cells separated by sheets of basal lamina (Lanzavecchia, '79; Bresciani, '91). Although similarity of architecture suggests that force generation also should be similar, Nematomorpha differ from nematodes in fundamental ways including their lack of a dorsal nerve cord (Cham et al., ' 83 ). The cuticular structure is quite different in detail from that of nematodes, and Valvassori et al. ('81) state that the sarcomeres have a completely different ultrastructure. There appear to be no descriptions of the motor behavior in Nematomorpha.

Many features of Mermis are shared by other mermithid genera (Kaiser, 1991): including the extremely threadlike body, 
smooth cuticle containing two layers of crossed helical fibers, six instead of eight muscle bands, a parasitic larval stage which absorbs nutrients through its cuticle, nonfeeding adults, and a greatly modified digestive tract for food storage. However, only in Mermis and Pheromermis, are the hosts infected by ingestion of eggs; in the other genera, second stage larvae actively invade the host. Only in Mermis nigrescens is the adult female specialized for navigating through vegetation. Larvae of other nematodes, including some Mermithidae, can climb over vegetation; however, their unusual length, makes female Mermis the only forms of nematodes that can bridge major discontinuities in the environment. Toward this end, nematode nictating behavior has become adapted in $\mathrm{Mer}$ mis for the search for new stems of vegetation not continuous with the surface being traversed. Moving over soil and climbing vegetation appears to be associated with the need to migrate from its over wintering site in soil to distribute eggs among the terminal leaves and shoots on which grasshoppers feed.

Locomotion in Mermis is seen to have a number of unique features. Mermis forms contact sites alternating with aerial curves; crawling with body waves elevated above the substratum is very rare. It is unlike "typical" nematode locomotion in which the dorsoventral waves lie in plane of the surface film and the worms travel on their sides; nor does it reflect any of the special modes of nematode locomotion (Stauffer, '24; Reed and Wallace, '65; Wallace, '59, '68; Seymour, '73; Keller and Falkovits, '83; Dobrolyukov, '86; Lee and Biggs, '90; Niebur and Erdos, '91). Adult female Mermis do not move by the classical undulant pattern of other nematodes (Gray and Lissmann, '64) or swimming snakes (Gray and Lissmann, '50), in which the waveform along the body is controlled as a single propulsive unit. The localized control over curvature observed for Mermis is not evident in Caenorhabditis elegans, nor has it been reported in other nematodes described.

\section{External force transmission}

A major question is how a propulsive force can be applied to substrate at the contact sites. The force transmission to a lacing site is similar to that utilized in propelling a snake past a single peg (Gray and Lissmann, '50; Gasc et al., '89; Gans, '94). The body bends around a fixed object such as a felt fiber or a grass stalk. The local curvature is asymmetrical; it attains a maximum (small- est radius) just anterior to the contact point and decreases posteriorly to it (Figs. 9, 10). As already demonstrated by Gray ('53) and used much earlier in the engineering socalled cam follower design (Ham et al., '58; Shigley, '61), a movable track will slide past a fixed resistance site so that travel is from a zone of small curvature to one of large curvature. In this interpretation, the body of the worm represents a muscularly deformable track that will travel past the fixed lacing site. The body will move in the direction that decreases the curvature at the contact. As the zone of muscular contraction creating the small localized curvature moves posteriorly along the body, there is continual forward sliding of the body.

The velocity with which the worm then slides past a site reflects a number of factors: the force applied to the site, forces transmitted axially from other sites, the momentum of the traveling object, and the friction at the site. The small mass of a worm $(5-15 \mathrm{mg})$ and low velocity indicate that momentum will be negligible relative to the potentially large forces of surface tension. The net force propelling a given segment of the body must be the sum of varying forces then being applied to the several individual contact points anterior and posterior to the region.

The waves do not obviously relate to the pattern of propulsion. The shape of such loops is even more misleading whenever the animals cannot fix the body at lacing sites, whereupon the contact points slide, the wave form changes, and the positions of force application shift among successful lacing sites. The body passes continuously past a contact site or through the path of an aerial curve, but the velocity can vary between regions. For each pair of lacing (force-transmitting) sites, the length of body between the sites and consequently the shape of the aerial segment represents a balance between the velocities of travel through the anterior and posterior sites. If the velocities differ, the shape will change. If the leading velocity is greater than the following one, the body loop will shrink and may shift from a compressive to a tensile state. If the reverse is true, the body will be under compression and the loop will increase in size. Additional loops may form as more of the body is shifted into the interval; the length of stiffened body loaded in compression will first of all deflect from the line connecting the lacing sites and next twist to form a new helical loop. These external manifestations of 
forces raise questions as to internal force production and coordination.

A body segment in one of the curves between contact sites will be in compression if the axial forces pushing it from behind exceed the axial forces pulling it forward. Evidence of this occurs when the tip is poked against a felt surface during lacing: the size of the loop in the body increases behind this point (Fig. 7a). The axial forces being transmitted by the stiff body derive from forces applied at one or more posterior contact sites. If, later, force is applied at the lacing site, the loop is diminished as tension develops in the same segment.

\section{Muscle architecture}

The locomotor muscles of the various nematodes for which we have descriptions all have oblique striations. This pattern may be geometrically confusing, which led to old arguments about whether obliquely striated muscle represented twitch or slow muscle (Toida et al., '75; Lanzavecchia, '77; Hoyle, '83). There are also differences in the way the contractile regions are packed within the cells, e.g., between coelomyarian and platymyarian muscle types (Hope, '69; Bird, '71), differences that may reflect the diameter of the worms. However, there is an overwhelming number of common denominators for obliquely striated muscle. Whatever the cell types, shapes and attachments, the myofilaments of the locomotor muscles always lie in parallel to the long axis of the worm and define the direction of shortening. The contractile region is clearly striated in that it has repeated sarcomeres with thick and thin filaments that slide past each other in shortening. The sarcomeres are staggered relative to each other, with adjacent sarcomeres advanced so that they lie at $\sim 6^{\circ}$, rather than $90^{\circ}$, to the longitudinal axis. The staggering produces the characteristic oblique striation observed in sections parallel to the cuticle in Caenorhabditis, or parallel to the intercellular sheets of basal lamina in Ascaris and Mermis. The myofilaments are longer than their equivalents in vertebrates (Rosenbluth, '67; Waterston and Francis, '85).

In another distinction from the vertebrate model, the contractile forces developed in the myofilaments are not transferred at the ends of the fibers to connective tissues and tendons. Instead, the forces are transmitted laterally to the cell membrane and a fibrous basal lamina. This lateral connection is the primary difference between oblique-striated and typical vertebrate cross-striated muscle.

The combination of lateral attachment and staggering of the myofilaments allows the worm to engage in very continuous, yet locally adjustable, shortening of the attachment sites, i.e. the longitudinal strips of cuticle. This potentially provides highly localized control of the shape and movement of the cuticular envelope. It should permit bending with minimal risk of major buckling of the cuticular surface. Thus it compensates for the absence of other skeletal structures.

\section{Cuticular structure}

The cuticle of Mermis has several features unique among nematodes that may be related to the unusual locomotory motion. Crossed helical fiber layers are found in some other nematodes, e.g., in Ascaris (Bird, '71). However, in Mermis and other mermithids, this trellislike structure lies in the outer cuticle rather than near the inner surface of the cuticle near the muscles. Fiber crossing angles in Mermis range from $111^{\circ}$ to $122^{\circ}$ in wholemounts, slightly larger than the angle of $110^{\circ}$ found by Lee ('70). A crossing angle of $110^{\circ}$ corresponds approximately to the critical spiral angle of $54.7^{\circ}$ for a spiral-wrapped hydroskeleton for which there is no change in volume or pressure with muscle-induced shortening; pressure would increase with shortening at the considerably larger angle, $150^{\circ}$, found for adult Ascaris (Harris and Crofton, '57). At the crossing angles observed in Mermis, a crossed helical structure approaches the functional limit of a spiral wrapped hydroskeleton (O'Grady, '83) and it is doubtful that it would provide the force necessary to restore the effect of muscular contraction. However, cutting of the cuticle evidently releases an internal pressure. The thick fibrous basal zone may provide the needed anisometric elasticity, and it is also better located nearer the muscle layer. This network of many smaller and shorter fibers is arrayed differently than in the equivalent layer of other nematodes. The cuticle of Mermis also lacks struts in the median zone, which in many nematode species provides a compliant connection between the basal and cortical layers (Inglis, '64; Wright, '91). However, the spongy underlying material of the median layer of Mermis may serve this purpose.

\section{Stiffness and internal forces}

Anesthetized Mermis and Ascaris are limp, yet, Mermis can stiffen short sections of its 
body so that these may be pushed along by forces originating at posterior contact sites. Such stiffening is observed whenever Mermis is poking the felt substrate during lacing. Also, in the pendulum platform experiment, the body is stiffened so that the initial contact of the head deflects the platform away from the fixed site on which the trunk of the worm rested. Further, Mermis can cantilever up to a third of its length as during head elevation bouts or nictating. Thus its very thin body not only withstands a limited amount of longitudinal compression, but it can also resist bending. This observation is truly remarkable because the long-column compression ratio to which the cantilevered portion is exposed is above $75(30 \mathrm{~mm} />.4$ $\mathrm{mm}$ ), well within the buckling range. The lack of long-column buckling with force application at the ends of such an elongate column needs an explanation as it is at the limits of technological capability.

Alexander ('87) has predicted that cuticles with helical fibers are under tension when the body is straight and lose strain energy when it bends, and therefore nematodes should normally be bent. However, Mermis straightens if anesthetized by immersion in a saturated solution of phenoxypropenol in saline. Also, Ascaris straightens whenever its muscles are relaxed by an injection of GABA (Burr, unpub. obs.).

What, then, stiffens the body of Mermis so that it can transmit collateral forces without buckling? Three nonexclusive possibilities exist. The first is that stiffness is due to a hydrostatic mechanism, the second assumes it to be inherent in the thick cuticle, and the third is that it is due to muscle stiffness. The occurrence of a hydrostatic component is reflected by the corrugation of the cuticle whenever the animal is shortened by dehydration. A component due to thick cuticle is indicated by the capacity of the dehydrated animal to move by bending. That the musculature contributes a large component to stiffness is shown by the limpness of anesthetized Mermis.

The thick-walled cuticle is clearly flexible enough to allow bending. Its internal meshwork of small fibers and helical crossing giant fibers will stiffen it locally and the internal lateral ridges may stiffen it further to restrict bending to the lateral plane. However, these structures and hydrostatic pressure provide only a fraction of the stiffness observed in unanaesthetized worms and are insufficient to prevent buckling during longcolumn compression.

It is clear that the body-wall muscle must provide most of the needed stiffness. Further, the structure of obliquely striated muscles provides a unique mechanism for applying bending couples over long distances without causing buckling. Because the longitudinal force required for cantilevering is applied locally, rather than at the ends of a muscle band, the required compressive force is distributed over continuous short local regions of the cuticle. In effect, the longcolumn compression ratio is reduced to a length that can be accommodated by the local cuticle without buckling.

Our finding that Mermis is able continuously to maintain a bending couple at a local site of force application while its body glides by is unique for the nematodes studied. This ability demands a control of the body muscles unlike that for classical nematode locomotion. Rather than being coordinated as one unit by signals originating in the anterior or posterior end, the muscles must be controlled locally, probably with local sensory feedback. Obliquely striated muscle fibers are well suited to the formation of local bending couples. Our evidence for a large number of motor neurons, perhaps two orders of magnitude above that of Ascaris or Caenorhabditis, suggests that the motor system of Mermis would appear to be capable of local control.

The locomotor mechanism appears to incorporate several behavioral subroutines that each deserve independent study. The cues for lacing, and for nictating need to be established, as do those that establish the relative magnitude of force application and velocity at each of the several lacing sites. Locomotion in a three-dimensional medium such as soil has yet to be examined in nematodes. One could look for similar locomotor behavior and mechanisms in other extremely elongate worms, such as other mermithids and in nematomorphs.

\section{ACKNOWLEDGMENTS}

This study was reported in part at the December 1992 Annual Meeting of the American Society of Zoologists. The work was supported by a grant from the Leo Leeser Foundation for Tropical Biology (to C.G.) and from the Natural Sciences and Engineering Research Council of Canada (to A.H.J. B.). The authors are grateful for the assistance of Gwen Bollerup for collection of Mermis, Ed North with some of the behavioral observa- 
tions, Hella Prochaska with the electron microscopy, Jennifer Wu with preparation of the micrographs, and Elizabeth Carefoot with the artwork.

\section{LITERATURE CITED}

Alexander, R. McN. (1987) Bending of cylindrical animals with helical fibres in their skin or cuticle. J. Theor. Biol. 124:97-110.

Bird, A.F. (1971) The Structure of Nematodes. London: Academic Press.

Bird, A.F. and J. Bird (1991) The Structure of Nematodes, 2nd ed. San Diego: Academic Press.

Bresciani, J. (1991) Nematomorpha. In F.W. Harrison and E.E. Ruppert (eds): Microscopic Anatomy of Invertebrates, Vol. 4. Aschelminthes. New York: Wiley-Liss, pp. 197-218.

Burr, A.H. (1985) The photomovement of Caenorhabditis elegans, a nematode which lacks ocelli. Proof that the response is to light and not radiant heating. Photochem. Photobiol. 41:577-582.

Burr, A.H.J., D.K. Eggleton, R. Patterson, and J.T. Leutscher-Hazelhoff (1989) The role of hemoglobin in the phototaxis of the nematode Mermis nigrescens. Photochem. Photobiol. 49:89-95.

Burr, A.H.J. and F.I. Harosi (1985) Naturally crystalline hemoglobin of the nematode Mermis nigrescens. Biophys. J. 47:527-536.

Burr, A.H.J. and C.P.F. Babinszki (1990) Scanning motion, ocellar morphology and orientation mechanism in the phototaxis of the nematode Mermis nigrescens. J. Comp. Physiol. A 167:257-268.

Burr, A.H.J., C.P.F. Babinszki, and A.J. Ward (1990) Components of phototaxis of the nematode Mermis nigrescens. J. Comp. Physiol. A 167:245-255.

Chalfie, M. and J. White (1988) The nervous system. In W.B. Wood (ed): The Nematode Caenorhabditis elegans. Cold Spring Harbor, NY: Cold Spring Harbor Laboratory, pp. 337-391.

Chalfie, M., J.E. Sulston, J.G. White, E. Southgate, J.N. Thomson, and $S$. Brenner (1985) The neural circuit for touch sensitivity in C. elegans. J. Neurosci. 5:956-964.

Cham, S.A., M.K. Seymour, and D.J. Hooper (1983) Observations on a British hairworm, Parachordodes wolterstorffii (Nematomorpha: Gordidae). J. Zool. (Lond,) 199:275-285.

Christie, J.R. (1937) Mermis subnigrescens, a nematode parasite of grasshoppers. J. Agric. Res. 55(5):353-364.

Cobb, N.A. (1926) The species of Mermis, a very remarkable group of nemas infesting insects. J. Parasitol. 8:66-72.

Cobb, N.A. (1929) The chromatropism of Mermis subnigrescens, a nemic parasite of grasshoppers. J. Wash. Acad. Sci. 19:159-166.

Crofton, H.D. (1971) Form, Function and Behaviour. In B.M. Zuckerman, W.F. Mai, and R.A. Rohde (eds): Plant Parasitic Nematodes, Vol. 1. New York: Academic Press, pp. 83-113.

Croll N.A. (1970) The Behavior of Nematodes: Their Activity, Senses and Responses. London: Edward Arnold.

Denny, M.W. (1993) Air and Water: The Biology and Physics of Life's Media. Princeton, NJ; Princeton University Press.

Dobrolyukov, A.I. (1986) The mechanism of locomotion of some terrestrial animals by traveling waves of deformation. J. Theor. Biol. 119:457-466.

Francis, R. and R.H. Waterston (1991) Muscle cell attachment in Caenorhabditis elegans. J. Cell Biol. 114:465479.
Gans, C. (1986) Locomotion of limbless vertebrates: Pattern and evolution. Herpetologica 42(1):33-46.

Gans, C. (1994) Approaches to the evolution of limbless locomotion. Cuadernos de Herpetol. 8(1):12-17.

Gasc, J.-P., D. Cattaert, C. Chasserat, and F. Clarac (1989) Propulsive action of a snake pushing against a single site: its combined analysis. J. Morph. 201:315329

Gray, J. (1953) Undulatory propulsion. Quart. J. Microsc. Sci. 94(4):551-578.

Gray, J.E. and H. Lissmann (1950) The kinetics of locomotion of the grass snake. J. Exp. Biol. 26:354-367.

Gray, J.E. and H. Lissmann (1964) The locomotion of nematodes. J. Exp. Biol. 41:135-154.

Ham, C.W., E.J. Crane, and W.L. Rogers (1958) Mechanics of Machinery, 4th ed. New York: McGraw-Hill.

Harris, J.E., and H.D. Crofton (1957) Structure and function in nematodes: internal pressure and cuticular structure in Ascaris. J. Exper. Biol. 34:116-130.

Hope, W.D. (1969) Fine structure of the somatic muscles of the free-living marine nematode Deontostoma californicum Steiner and Albin, 1933 (Leptosomatidae). Proc. Helminthol. Soc. 36:10-29.

Hoyle, G. (1983) Muscles and Their Neural Control. New York: Wiley-Interscience.

Hyman, L.H. (1951) The Invertebrates. Vol. 3. Acanthocephala, Aschelminthes and Entoprocta. The Pseudocoelomate Bilateria. New York: McGraw-Hill.

Inglis, W.G. (1964) The structure of the nematode cuticle. J. Zool. (Lond.) 143:465-502.

Johnson, C.D., and A.O.W. Stretton (1980) Neural control of locomotion in Ascaris: Anatomy, electrophysiology and biochemistry. In B.M. Zuckerman (ed): Nematodes as Biological Models, Vol. 1. New York: Academic Press, pp. 159-195.

Kaiser, H. (1991) Terrestrial and semiterrestrial Mermithidae. In W.R. Nickle (ed): Manual of Agricultural Nematology. New York: Marcel Dekker, pp. 899-965.

Keller, J.B. and M.S. Falkovits (1983) Crawling of worms. J. Theor. Biol. 104:417-492.

Lanzavecchia, G. (1977) Morphological modulations in helical muscles (Aschelminthes and Annelida). Internat. Rev. Cytol. 51:133-186.

Lanzavecchia, G. (1979) Three-dimensional reconstruction of the contractile system of the Nematomorpha muscle fiber. J. Ultrastruct. Res. 66:201-223.

Lee, D.L. (1970) The ultrastructure of the cuticle of adult female Mermis nigrescens (Nematoda). J. Zool. (Lond.) 161:513-518.

Lee, D.L. and H.J. Atkinson (1977) The Physiology of Nematodes, 2nd ed. New York: Columbia University Press.

Lee, D.L. and W.D. Biggs. (1990) Two- and threedimensional locomotion of the nematode Nippostrongylus brasiliensis. Parasitol, 101:301-308.

Maggenti, A.R. (1983) Nematode higher classification as influenced by species and family concepts. In A.R. Stone, H.M. Platt, and L.F. Khalil (eds): Concepts in Nematode Systematics. London: Academic Press, pp. 25-40.

Niebur, E. and P. Erdos (1991) Theory of the locomotion of nematodes. Dynamics of undulatory progression on a surface. Biophys. J. 60:1132-1146.

O'Grady, R.T. (1983) Cuticular changes and structural dynamics in the fourth-stage larvae and adults of Ascaris suum Goeze, 1782 (Nematoda: Ascaridaidea) developing in swine. Canad. J. Zool. 61:1293-1303.

Rankin, C.H. (1991) Interactions between two antagonistic reflexes in the nematode Caenorhabditis elegans. J. Comp. Physiol., A 169:59-67. 
Reed, E.M. and H.R. Wallace (1965) Leaping locomotion by an insect parasitic nematode. Nature, Lond. 206: 210-211.

Rosenbluth, J. (1965) Ultrastructural organization of obliquely striated muscle fibers in Ascaris lumbricoides. J. Cell. Biol, 25:495-515.

Rosenbluth, J. (1967) Obliquely striated muscle. III. Contraction mechanism of Ascaris body muscle. J. Cell. Biol. 34: 15-33.

Seymour, M.K. (1973) Motion and the skeleton in small nematodes. Nematologica 19:43-48.

Seymour, M.K. (1983) Some implications of helical fibers in worm cuticles. J. Zool. (Lond.) 199:287-295.

Shigley, J.E. (1961) Theory of Machines. New York: McGraw-Hill.

Stauffer, H. (1924) Die Lokomotion der Nematoden. Beiträge zur Kausalmorphologie der Fadenwürmer. Zool. Jahrb. (Syst.) 49:1-118.

Stretton, A.O.W. (1976) Anatomy and development of the somatic musculature of the nematode Ascaris. J. Exp. Biol. 64:773-788.

Stretton, A.O.W., R.M. Fishpool, E. Southgate, J.E. Donmoyer, J.P. Walrond, J.E.R. Moses, and I.S. Kass (1978) Structure and physiological activity of the motoneurons of the nematode Ascaris. Proc. Natl. Acad. Sci. 75:3493-3497.

Streu, H.T., W.R. Jenkins, and M.T. Hutchinson (1961) Nematodes associated with carnations. New Jersey Agric. Exp. Sta. Rutgers Bull. 800.

Sulston, J.E. and H.R. Horvitz (1977) Post-embryonic cell lineages of the nematode Caenorhabditis elegans. Develop. Biol. 56:110-156.

Thomas, J.H. (1990) Genetic analysis of defecation in Caenorhabditis elegans. Geneties 124:855-872.

Toida, N., H. Kuriyama, N. Tashiro, and Y. Ito (1975) Obliquely striated muscle. Physiol. Revs. 55:700-756.

Valvassori, R., M. de Equileor, and G. Lanzavecchia (1981) Studies on the helical and paramyosinic muscle.
VIII. Ultrastructural analysis of body wall muscles from Mermis sp. J. Ultrastruct. Res. 76:82-88.

Wallace, H.R. (1959) Movement of eelworms in water films. Ann. Appl. Biol. 47(2):366-370.

Wallace, H.R. (1968) Dynamics of nematode movement. A. Rev. Phytopath. 6:91-114.

Walrond, J.P., I.S. Kass, A.O.W. Stretton, and J.E. Donmoyer (1985) Identification of excitatory and inhibitory motoneurons in the nematode Ascaris by electrophysiological techniques. J. Neurosci. 5:1-8.

Waterston, R.H. (1988) Muscle. In W.B. Wood (ed): The Nematode Caenorhabditis elegans. Cold Spring Harbor, NY: Cold Spring Harbor Laboratory, pp. 281-335.

Waterston, R.H. and G.R. Francis (1985) Genetic analysis of muscle development in Caenorhabditis elegans. Trends Neurosci. 8:270-276.

Wharton, D.A. (1986) A Functional Biology of Nematodes. London: Croon Helm

White, J.G., E. Southgate, J.N. Thomson, and S. Brenner (1976) The structure of the ventral nerve cord of Caenorhabditis elegans. Phil. Trans. Roy. Soc. Lond. B 275:327-348.

White, J.G., E. Southgate, J.N. Thomson, and S. Brenner (1986) The structure of the nervous system of Caenorhabditis elegans. Phil. Trans. Roy. Soc. Lond. B 314:1-340.

Wright, K.A. (1987) The nematode's cuticle-its surface and the epidermis: Function, homology, analogy-a current consensus. J. Parasitol. 73:1077-1083.

Wright, K.A. (1991) Nematoda. In F.W. Harrison, and E.E. Ruppert (eds): Microscopic Anatomy of Invertebrates, Vol. 4. Aschelminthes. New York: Wiley-Liss, pp. 111-195.

Zapotsky, J.E. (1971) The cuticular ultrastructure of Paragordius varius (Leidy, 1851) (Gordioidea: Chordodidae). Proc. Helminthol. Soc. Wash. 38:228-236. 